\title{
Ingestão de pescados e repercussões no recém-nascido: estudo-piloto
}

\author{
Fish intake and repercussions in the newborn: pilot study
}

\author{
Norma Elizabete Rattes, ${ }^{1}$ Mauricio Afonso Vericimo, ${ }^{1}$ Glaucia Macedo de Lima, ${ }^{1}$ Adauto Dutra Barbosa ${ }^{1}$ e Israel \\ Figueiredo Jr. ${ }^{1}$
}

Universidade Federal Fluminense

\section{Palavras-chave:}

Peso ao nascer; Ingestão de alimentos; Gravidez; Recém-nascido; Recém-nascido prematuro; Peixes.

\section{Resumo}

Introdução: o peixe foi e sempre será uma importante fonte de proteína na alimentação. Objetivo: verificar se a quantidade de peixe ingerida durante uma gravidez normal pode influenciar na idade gestacional e peso ao nascimento. Métodos: estudo-piloto retrospectivo com análise de dados gerados e armazenados em banco de dados após aplicação de questionário estruturado e avaliação de prontuários de puérperas e seus recém-nascidos. Foram recolhidos elementos relacionados à gestação, ao recém-nascido e informações referentes à ingestão de peixes e frutos do mar. Após a aplicação dos critérios de exclusão, foi testada a relação entre variáveis categóricas com o teste de qui-quadrado e Kruskal-Wallis para as variáveis contínuas, todos com significância de 0,05. Resultados: entre 309 binômios mãe-filho 40 foram selecionados, sendo que a maioria morava em Niterói (38/40). A média de idade foi de 24,8 anos e o número médio de consultas de pré-natal foi de 5,7. Nenhum recém-nascido precisou de reanimação e a média da idade gestacional e do peso ao nascimento foram, respectivamente, 39,2 semanas e 3.224,2 gramas. A maioria ingeriu pescados esporadicamente $(n=11 ; 32,5 \%)$. Sete $(17,5 \%)$ declararam ingerir peixe duas vezes ou mais por semana. A idade gestacional de nascimento foi significativamente diferente $(p=0,012)$ quando comparadas às mães que referiram baixa e alta ingestão de peixe. Conclusões: a maioria das mulheres analisadas não ingeriu a quantidade recomendada de peixe durante a gravidez. O consumo de peixe não influenciou significativamente no peso neonatal, entretanto a idade gestacional de nascimento foi menor no grupo que referiu maior ingesta de pescados.

\section{Keywords:}

Birth weight; Eating; Pregnancy; Infant, newborn; Infant, premature; Fishes.

\footnotetext{
Abstract

Introduction: fish has always been an important source of protein for humans. Objective: to ascertain whether fish intake during pregnancy can influence birth weight and gestational age at delivery. Methods: retrospective pilot study of a database compiled after administration of a structured questionnaire and review of medical records of postpartum women and their infants. Information was collected on variables related to pregnancy, neonatal outcomes, and maternal fish and seafood intake. After application of the exclusion criteria, associations between variables were evaluated with the chi-square test (categorical variables) or Kruskal-Wallis test (continuous variables). The significance level was set at 0.05. Results: from a sample of 309 mother-child pairs, 40 were selected. Most $(n=38)$ lived in the municipality of Niterói. The mean maternal age was 24.8 years, and the mean number of antenatal care visits was 5.7. No neonates required resuscitation. The mean gestational age and weight at birth were 39.2 weeks and 3,224.2 g, respectively. Most women reported only sporadic fish intake $(n=11 ; 32.5 \%)$. Seven $(17.5 \%)$ reported fish intake two or more times per week. Gestational age at birth was significantly different ( $p=0.012$ ) when compared to women who reported low vs. high frequency of fish intake. Conclusions: most women included in this study did not ingest the recommended amount of fish during pregnancy. Fish intake had no significant influence on birth weight; however, gestational age at birth was lower in the group that reported higher levels of fish intake.
} 


\section{INTRODUÇÃO}

Deficit e excessos alimentares antes e durante a gravidez estão associados a repercussões perinatais. ${ }^{1} \mathrm{~A}$ alimentação adequada contribui para o êxito da gravidez e o peixe é uma fonte importante de proteína.

Os efeitos benéficos da ingestão de pescados e frutos do mar se devem à presença de proteínas de altíssima qualidade, pequena quantidade de gordura saturada e nutrientes essenciais (iodo, ferro, colina e selênio), além de ácidos graxos poli-insaturados de cadeia longa, especialmente ômega- $3 .^{2}$ A ingestão de peixe durante a gestação também está positivamente associada ao desenvolvimento cognitivo na infância ${ }^{3}$ e vem sendo associado a um padrão normal de consumo de peixe em torno de 340 gramas por semana. ${ }^{4}$

Revisão sistemática recente mostrou que a suplementação de ácido graxo poli-insaturado de cadeia longa n-3 na gestação esteve associada à diminuição do risco de parto pré-termo e a um modesto aumento no peso de nascimento. ${ }^{5}$

Entretanto, dietas inadequadas durante a gravidez podem contribuir para nascimento prematuro e restrição de crescimento, ${ }^{1}$ aumentam o risco de diabetes gestacional ${ }^{6}$ e depressão puerperal (não comprovada), ${ }^{7}$ e estão associadas a diversos tipos de intoxicações. ${ }^{8-10}$

O crescimento fetal intraútero em mulheres que consomem peixes e frutos do mar continua motivando os pesquisadores. Existem poucos trabalhos brasileiros sobre os benefícios e riscos do consumo de pescados para a saúde humana, ${ }^{11}$ incluindo a ingestão durante a gestação em mulheres sem problemas e possíveis repercussões fetais.

O objetivo deste trabalho foi verificar se o consumo de peixe influenciou o peso e a idade gestacional ao nascimento, além de definir se a quantidade ingerida estava adequada para o período gestacional.

\section{MÉTODO}

Esse módulo descreve os elementos necessários para o entendimento da pesquisa, levando-se em consideração as etapas e os processos estatísticos aplicados.

\section{DESENHO DO ESTUDO}

Estudo-piloto retrospectivo, transversal, elaborado a partir da verificação de dados de prontuários médicos armazenados, referentes ao projeto aprovado pelo Comitê de Ética em Pesquisa da Universidade Federal Fluminense - Certificado de Apresentação para Apreciação Ética: 0167.0.258.000-08, vinculado à pesquisa do Laboratório de Imunobiologia da universidade.
Além do recolhimento em prontuários, um questionário estruturado foi utilizado para coleta específica desses dados em puérperas nos dias subsequentes ao parto em unidades hospitalares.

\section{CENÁRIO E PARTICIPANTES}

A coleta de dados foi realizada por um dos pesquisadores em unidades públicas perinatais na cidade de Niterói, Rio de Janeiro, Brasil, entre janeiro de 2009 e dezembro de 2011.

Concordaram em participar do estudo 309 puérperas que estiveram internadas nas maternidades avaliadas durante o período de coleta de dados. Todas assinaram o termo de consentimento. Os dados selecionados para montagem do banco de dados foram retirados de prontuário médico, pareceres do serviço social e anotações de enfermagem, além da informação colhida com a própria puérpera. Foram incluídas na análise todas as pacientes atendidas em unidade perinatal de baixo risco $(\mathrm{n}=139)$. Foram excluídas as que tinham idade menor que 18 (n $=9$ ) e maior que 40 anos $(n=3)$, as que apresentaram intercorrências no pré-natal $(n=59)$ (situações agudas como doenças hipertensivas, infecção do trato urinário, ameaça de parto prematuro, além de uso de fumos ou ingestão de substâncias impróprias e situações crônicas com possíveis patologias prévias), aquelas que tiveram seus recém-nascidos com problemas no berçário $(\mathrm{n}=6)$ e as que tiveram partos cesarianos $(n=22)$. Ao final, a amostra foi composta por 40 binômios mãe-filho que não apresentavam qualquer tipo de problema.

\section{VARIÁVEIS}

As variáveis categóricas estudadas foram município de moradia, número de consultas no pré-natal, sexo do recém-nascido, frequência de consumo de peixe e frutos do mar, tipo de peixe e ingestão ou não de peixe cru. Para avaliação do tipo de peixe consumido foram recolhidas as quatro primeiras preferências, e esse bloco de informações recebeu análise específica de variável com múltiplas respostas. Já as variáveis contínuas estudadas foram idade maternal, número de consultas no pré-natal, idade gestacional, peso ao nascimento, Apgar no $5^{\circ}$ minuto e dias de internação.

\section{FONTES DE DADOS/MENSURAÇÕES}

No questionário, as puérperas foram interrogadas se ingeriam peixe cru e a frequência de ingestão de peixe foi classificada como diária, semanal $(6,5,4,3,2$, ou $1 \times /$ semana), mensal $(3,2,1 \times /$ mês) ou esporádica (consumo $<1 \times /$ mês $)$. 
Considerou-se baixo peso ao nascer aquele menor que 2.500 gramas. Em ambas as unidades de nascimento as balanças eram digitais e sempre manipuladas por pessoal treinado em área perinatal. Para esta pesquisa foi levado em consideração o peso e a idade gestacional constantes na Declaração de Nascido Vivo. A idade gestacional foi convertida para dias de gestação para análise estatística e depois novamente recalculada em semanas para divulgação dos resultados. A gestação abaixo de 37 semanas foi considerada prematura. O recém-nascido foi classificado por percentil com base em uma curva de crescimento nacional, ${ }^{12}$ que levou em consideração o peso, a idade gestacional e o sexo.

\section{MÉTODOS ESTATÍSTICOS}

As variáveis contínuas foram exploradas levando-se em consideração média, mediana e desvio-padrão. Conforme Corres e colaboradores, foram caracterizadas como tendo uma ingestão grande de peixe as mulheres que referiram ingerir a proteína duas vezes ou mais por semana. ${ }^{13}$ As puérperas foram divididas em três grupos: as que referiam ingerir peixe e frutos do mar duas vezes por semana ou mais ( $\geq 2 \times /$ semana); as que comiam abaixo de duas vezes por semana $(<2 \times /$ semana $)$; e as que não comiam peixe $(0 \times /$ semana). Os grupos foram comparados em relação ao peso e à idade gestacional ao nascer.

O pacote estatístico SPSS ${ }^{\circledR}$ versão 17.0 (SPSS Inc., Chicago) foi utilizado para a realização das análises. A análise de múltiplas respostas foi utilizada para caracterização dos peixes e frutos do mar nos dois grupos. Posteriormente, foi criada uma tabela de contingência entre frequência de ingestão de peixe e tipo de peixe para verificar a existência de diferenças entre as espécies de pescados nos dois grupos, a partir da aplicação do teste do qui-quadrado com teste exato de Fisher $(p<0,05)$.

Teste não paramétrico para mais de duas amostras independentes (teste de Kruskal-Wallis) foi utilizado, levando-se em consideração como variável de agrupamento à frequência de ingestão semanal $(\geq 2 \times 1$ semana, $<2 \times /$ semana e $0 \times /$ semana) e as variáveis-testes idade materna, número de consultas no pré-natal, idade gestacional, peso ao nascimento, Apgar no $5^{\circ} \mathrm{mi}$ nuto e dias de internação. Todos os testes tiveram nível de significância de 0,05. O teste post-hoc de Bonferroni, a partir da análise linear generalizada (GzLM) com distribuição gama, foi a opção de ajuste, com a variável dependente, sendo a/o idade gestacional/peso e o fator fixo a frequência de ingestão $(0 / 1 / 2)$.

\section{RESULTADOS}

Dos 40 binômios mãe-filho analisados, a maioria (95,0\%) morava em Niterói (38/40). A idade média da puérpera foi de 24,8 (IC $95 \%$ de 22,9 a 26,8; desvio-padrão $=5,4$ ) e o número de consultas no atendimento pré-natal de 5,7 (IC 95\% de 5,1 a 6,2; desvio-padrão = 1,5).

Dos recém-nascidos avaliados, 55,0\% foram do sexo feminino $(22 / 40)$ e apenas um teve a idade gestacional abaixo de 37 semanas. A média de idade gestacional ao nascimento foi de 39,2 semanas (IC $95 \%$ de 39, 2 a 40,0; desvio-padrão $=1,1$ ) e o peso médio ao nascimento foi de 3.224,2 gramas (IC 95\% de 3.075,9 a 3.372,5; desvio-padrão $=425,0)$. Somente um recém-nascido apresentou peso abaixo de 2.500 gramas $(2.455 \mathrm{~g})$. Nenhum recém-nascido precisou de reanimação e foram pontuados com mediana de 9.0 no Apgar de $5^{\circ}$ minuto (IC $95 \%$ de 8,6 a 9,3; desvio-padrão $=1,0)$. Todos permaneceram entre dois e cinco dias no local do nascimento (média $=$ 2,6; IC 95\% de 2,2 a 2,9; desvio-padrão $=0,9$ ).

A maioria das puérperas $(32,5 \%)$ relatou ingerir esporadicamente pescados e frutos do mar (11/40) (Figura $1 \mathrm{~A})$. Sete $(17,5 \%)$ declararam ingerir peixe duas vezes ou mais por semana, e duas comiam peixe cru.

Foram citadas 33 espécies de peixe, com um total de 106 respostas válidas, a partir da análise de variável com múltiplas respostas. Os mais consumidos foram: crustáceos (Penaeus brasiliensis) - 23,6\% (25/106); sardinha brasileira (Brazilian sardine/Sardinella brasiliensis)

Tabela 1 - Teste de Kruskal-Wallis mostrando que só a idade gestacional apresentou diferença significativa entre as médias ranqueadas quando comparada entre os grupos com baixa, alta ou nenhuma ingestão de pescados

\begin{tabular}{lllllll}
\hline & \multicolumn{5}{c}{ Teste estatístico $\mathbf{a}^{\mathbf{a}, \mathbf{b}}$} \\
\hline & Idade materna & Consulta pré-natal & Idade gestacional & Peso & Apgar5 & Dias internação \\
\hline Chi-square & 154 & 2,072 & 7,688 & 2,237 & 1,554 &, 197 \\
df & 2 & 2 & 2 & 2 & 2 & 2 \\
Asymp. Sig. &, 926 &, 355 &, 021 &, 327 &, 460 &, 906 \\
\hline
\end{tabular}


Figura 1 - A) Frequência semanal/mensal da ingestão de pescados e frutos do mar pelas puérperas; e B) distribuição das idades gestacionais nos três grupos analisados: nenhuma; $<2 x_{;}>2 \times /$ semana

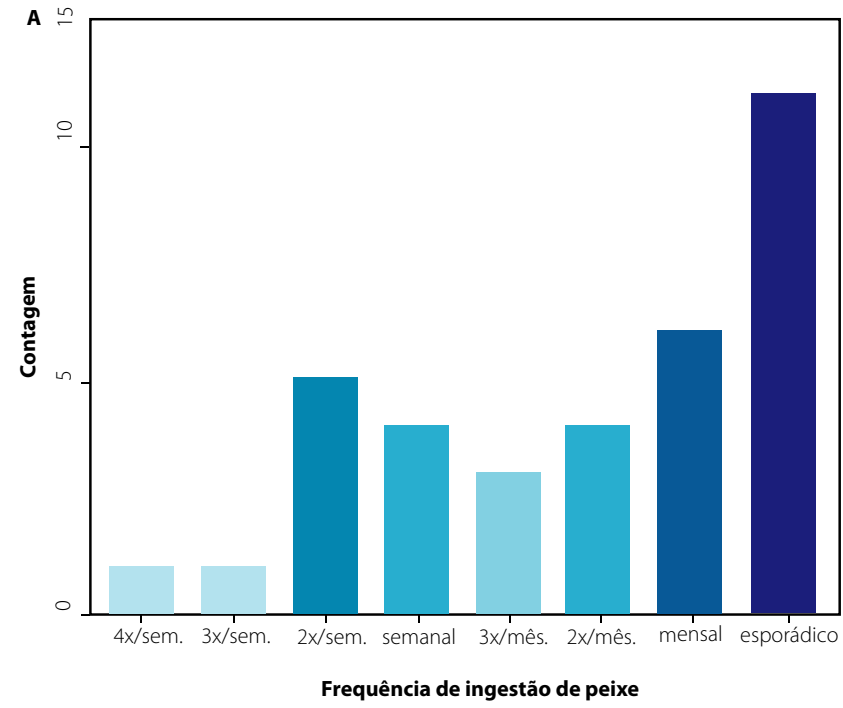

Fonte: Banco de dados ligado ao Instituto de Imunobiologia da UFF (2017).

- 18,9\% (20/106); e corvina (Whitemouth croaker/ Micropogonias furnieri) - 17,0\% (18/106).

Não houve diferenças significativas entre os três grupos em relação à presença ou não de seis consultas no pré-natal $(\mathrm{p}=0,112)$, hábito de ingestão de peixe cru ( $\mathrm{p}$ $=0,500)$, peso ao nascimento $(p=0,548)$ e sexo do recém-nascido $(p=0,890)$. A análise dos percentis não revelou diferenças significativas $(p=0,676)$. Também não houve diferença significativa $(p=0,100)$ entre a preferência nos grupos que ingeriam peixes e frutos do mar, sendo os crustáceos os mais comuns.

A idade gestacional distribuída graficamente mostrou medianas dos grupos em pontos diferentes, apesar de todos acima de 37 semanas (Figura 1B). Somente a idade gestacional demonstrou diferença significativa $(\mathrm{p}=$ 0,012 ) entre os grupos, tendo o teste post-hoc mostrado uma diferença significativa entre os grupos com consumo de peixe acima de duas vezes por semana e abaixo de duas vezes por semana.

\section{DISCUSSÃO}

Ficou evidente na pesquisa que as gestantes ingeriram a proteína de peixe de forma modesta, tendo aquelas com um maior consumo apresentado uma pequena diferença da idade gestacional ao nascimento, mesmo tendo praticamente todas as gestações chegado a termo. Esta análise, apesar de ter mostrado uma remota

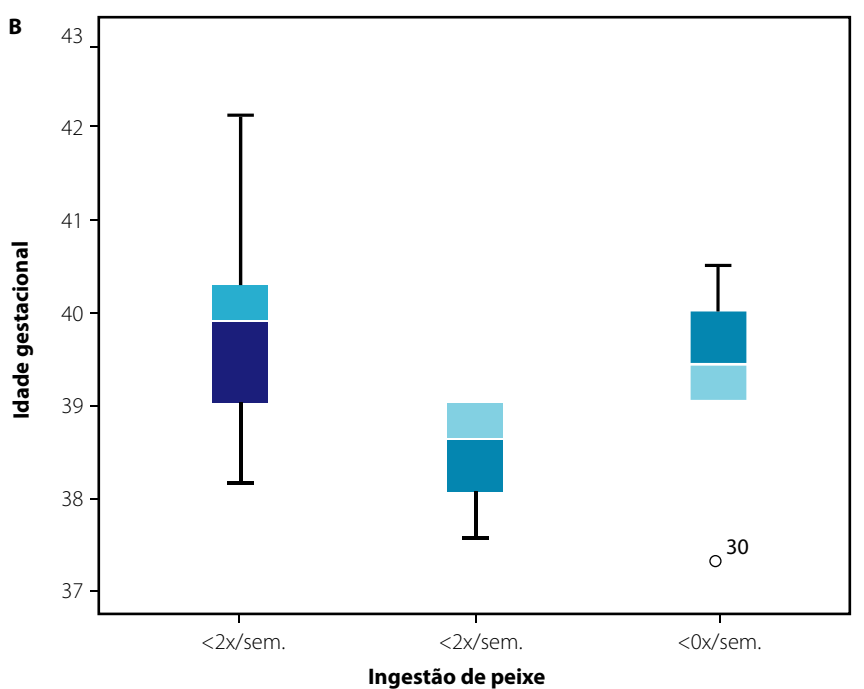

possibilidade de interferência da ingestão de peixe no tempo da gestação, vai de encontro a trabalho de grande porte no qual foi mostrado que a ingestão moderada de peixe durante a gestação esteve associada a uma menor chance de nascimento pré-termo. ${ }^{14}$

A opção por dieta pobre em peixes e frutos do mar durante a gestação já vem sendo descrita. A Agência Americana de Proteção Ambiental e de administração de drogas e alimentos após pesquisa demonstrou que a maioria das mulheres grávidas não comem muito peixe, com consequente ingestão inadequada de ácidos gordurosos. ${ }^{15}$ Esse dado é inquietante já que o peixe é uma excelente fonte proteica, recomendada pelo FDA, ao invés dos complementos com ômega-3. ${ }^{15}$ A preocupação vem diminuindo, já que trabalhos recentes mostram que uma alta aderência à dieta mediterrânea, modismo atual, tem significativamente menor risco no desenvolvimento de restrição de crescimento uterino para o peso, ${ }^{16-18}$ embora nessa amostra não se tenha encontrado diferença de peso estatisticamente significativa entre os grupos que comeram e não comeram peixe.

Algumas limitações fazem parte desse estudo, já que se tratou de um projeto-piloto, com aproveitamento de banco de dados já existente. Dada a natureza retrospectiva do estudo, algumas variáveis não puderam ser consideradas, por exemplo a paridade, pois o número de gestações acaba tendo alguma influência nos pesos dos fetos 
subsequentes. Como inicialmente não foi um trabalho que assumiu uma interface eminentemente nutricional, não houve a preocupação de congregar as gestantes em grupos de dietas para uma melhor análise. Não foram encontradas evidências nos prontuários e durante a entrevista de utilização de drogas lícitas e ilícitas, incluindo a nicotina, esta comprovadamente responsável por restrições de crescimento fetal. ${ }^{17}$

Apesar de caminhar com a literatura, reconhecendo que não existe associação entre uma grande ingestão de peixe e o crescimento fetal, Guldneret et al. ${ }^{19}$ sugerem que categorias de frutos do mar estão diferentemente associadas com os desfechos das gestações, sendo que o consumo de peixe seria benéfico ao crescimento fetal, enquanto o consumo de mariscos (shellfish) poderia gerar um decréscimo do tamanho fetal. Segundo Ramon e cols., ${ }^{20} \mathrm{O}$ consumo de duas porções por semana de grandes peixes oleosos esteve associado a um maior risco de ser pequeno para idade gestacional para o peso, enquanto o consumo de peixe magro geraria um menor risco de ser pequeno para o comprimento, quando em comparação com um consumo abaixo de uma porção por mês.

Apesar do pequeno número de casos analisados e da dificuldade de generalização, percebe-se que o tema deve continuar a ser alvo de pesquisas. Também pelo fato de o trabalho ter sido derivado do aproveitamento de um banco de dados pré-existente, outra limitação foi a ausência de dados sobre o estado nutricional da mãe antes da gestação e sobre o ganho ponderal durante a gravidez, o que também pode interferir nos resultados do crescimento fetal.

\section{CONCLUSÕES}

Conclui-se que a maioria das mulheres analisadas não ingeriu quantidade recomendada de peixe durante a gravidez e que a frequência de ingestão de peixe não interferiu significativamente no peso neonatal, mas influenciou na idade gestacional de nascimento do grupo estudado.

\section{REFERÊNCIAS}

1 Grieger JA, Grzeskowiak LE, Clifton VL. Preconception dietary patterns in human pregnancies are associated with preterm delivery. J Nutr. 2014;144(7):1075-80.

2 Deroma L, Valent F, Parpinel M, Barbone F. Comparison of seafood consumption in a group of Italian mother-child pairs. J Health Popul Nutr. 2013;31(4):455-61.

3 Deroma L, Parpinel M, Tognin V, Channoufi L, Tratnik J, Horvat M et al. Neuropsychological assessment at school-age and prenatal low-level exposure to mercury through fish consumption in an Italian birth cohort living near a contaminated site. Int J Hyg Environ Health. 2013;216(4):486-93.

4 Julvez J, Mendez M, Fernandez-Barres S, Romaguera D, Vioque J, Llop S et al. Maternal consumption of seafood in pregnancy and child neuropsychological development: a longitudinal study based on a population with high consumption levels. Am J Epidemiol. 2016;183(3):169-82.

5 Imhoff-Kunsch B, Briggs V, Goldenberg T, Ramakrishnan U. Effect of n-3 long-chain polyunsaturated fatty acid intake during pregnancy on maternal, infant, and child health outcomes: a systematic review. Paediatr Perinat Epidemiol. 2012;26(Suppl 1):91-107.

6 Bao W, Bowers K, Tobias DK, Hu FB, Zhang C. Prepregnancy dietary protein intake, major dietary protein sources, and the risk of gestational diabetes mellitus: a prospective cohort study. Diabetes Care. 2013;36(7):2001-8.

7 Miyake Y, Sasaki S, Yokoyama T, Tanaka K, Ohya Y, Fukushima W et al. Risk of postpartum depression in relation to dietary fish and fat intake in Japan: the Osaka Maternal and Child Health Study. Psychol Med. 2006;36(12):1727-35.

8 Mania M, Wojciechowska-Mazurek M, Starska K, Rebeniak M, Postupolski J. Fish and seafood as a source of human exposure to methylmercury. Rocz Panstw Zakl Hig. 2012;63(3):257-64.

9 Wantke F, Gotz M, Jarisch R. Histamine-free diet: treatment of choice for histamine-induced food intolerance and supporting treatment for chronic headaches. Clin Exp Allergy. 1993;23(12):982-5.

10 Shibata E, Ueda T, Akaike G, Saida Y. CT findings of gastric and intestinal anisakiasis. Abdom Imaging. 2014;39(2):257-61.

11 Fernades AC, Medeiros CO, Bernardo GL, Ebone MV, Di Pietro PF, Assis MAA, et al. Benefits and risks of fish consumption for the human health. Rev Nutr. 2012;25(2):283-95.

12 Pedreira CE, Pinto FA, Pereira SP, Costa ES. Birth weight patterns by gestational age in Brazil. An Acad Bras Ciênc. 2011;83(2):619-25.

13 Corres LF, Del Pozo MD, Aizpuru F. Prevalence of Anisakis simplex sensitisation in three Spanish areas, in relation to the fish intake rates. Relevance of Anisakis simplex allergy. Alergol Inmunol Clin. 2001;16:337-46.

14 Leventakou V, Roumeliotaki T, Martinez D, Barros H, Brantsaeter AL, Casas M et al. Fish intake during pregnancy, fetal growth, and gestational length in 19 European birth cohort studies. Am J Clin Nutr. 2014;99(3):506-16.

15 Wenstrom KD. The FDA's new advice on fish: it's complicated. Am J Obstet Gynecol. 2014;211(5):475-8.el.

16 Timmermans S, Steegers-Theunissen RP, Vujkovic M, den Breeijen H, Russcher H, Lindemans J et al. The Mediterranean diet and fetal size parameters: the Generation R Study. Br J Nutr. 2012;108(8):1399-409.

17 Chatzi L, Mendez M, Garcia R, Roumeliotaki T, Ibarluzea J, Tardón A et al. Mediterranean diet adherence during pregnancy and fetal growth: INMA (Spain) and RHEA (Greece) mother-child cohort studies. Br J Nutr. 2012;107(1):135-45

18 Brantsaeter AL, Birgisdottir BE, Meltzer HM, Kvalem HE, Alexander J, Magnus P et al. Maternal seafood consumption and infant birth weight, length and head circumference in the Norwegian Mother and Child Cohort Study. Br J Nutr. 2012;107(3):436-44.

19 Guldner L, Monfort C, Rouget F, Garlantezec R, Cordier S Maternal fish and shellfish intake and pregnancy outcomes: a prospective cohort study in Brittany, France. Environ Health. 2007;6:33.

20 Ramon R, Ballester F, Aguinagalde X, Amurrio A, Vioque $\mathrm{J}$, Lacasana $\mathrm{M}$ et al. Fish consumption during pregnancy, prenatal mercury exposure, and anthropometric measures at birth in a prospective mother-infant cohort study in Spain. Am J Clin Nutr. 2009;90(4):1047-55. 